\title{
LCM: Lightweight Communications and Marshalling
}

\author{
Albert S. Huang, Edwin Olson, David C. Moore
}

\begin{abstract}
We describe the Lightweight Communications and Marshalling (LCM) library for message passing and data marshalling. The primary goal of LCM is to simplify the development of low-latency message passing systems, especially for real-time robotics research applications.

Messages can be transmitted between different processes using LCM's publish/subscribe message-passing system. A platform- and language-independent type specification language separates message description from implementation. Message specifications are automatically compiled into language-specific bindings, eliminating the need for users to implement marshalling code while guaranteeing run-time type safety.

LCM is notable in providing a real-time deep traffic inspection tool that can decode and display message traffic with minimal user effort and no impact on overall system performance. This and other features emphasize LCM's focus on simplifying both the development and debugging of message passing systems. In this paper, we explain the design of LCM, evaluate its performance, and describe its application to a number of autonomous land, underwater, and aerial robots.
\end{abstract}

\section{INTRODUCTION}

A fundamental software design principle is that of modularity, which promotes maintainability, code re-use, and fault isolation [1]. A large robotic system, for example, can be decomposed into specific tasks such as data acquisition, state estimation, task planning, etc. To accomplish their tasks, modules must exchange information with other modules. With modern operating systems, it is convenient to map individual modules onto software processes that can be on the same or physically separate computational devices. This then transforms the task of information exchange into the well studied problem of interprocess communication.

In this paper, we describe a message passing system for interprocess communication that is specifically targeted for the development of real-time systems. Our approach is motivated by lessons from modern software practices, and places great emphasis on simplicity and usability from the perspective of a system designer. We call our system Lightweight Communications and Marshalling (LCM) to signify its functionality and its simplicity in both usage and implementation.

The single most notable attribute of mapping modules onto separate processes is that every module receives a separate memory address space. The introduction of this barrier provides a number of benefits; modules can be run on

Huang is at the Computer Science and Artificial Intelligence Laboratory at MIT, Cambridge, MA, USA. Olson is in the Computer Science and Engineering department at the University of Michigan, Ann Arbor, MI, USA. Moore is a software engineer at Dreamworks SKG, Inc. the same or different host devices, started and stopped independently, written in different programming languages and for different operating systems, and catastrophic failure of one module (e.g. a segmentation fault) does not necessarily impact another.

With this independence also comes isolation - sharing information between modules is no longer a trivial task. Module designers must carefully consider what information to share across modules, how to marshal (encode) that information into a message, how to communicate a marshalled message from one module to another, and how to un-marshal (decode) the message once received.

Although a message passing system introduces complexities that must be carefully managed, it also provides opportunities for analysis and introspection that may be invaluable to a developer. In particular, messages may be captured and analyzed by modules specifically designed to aid system development. Such modules might log messages to disk, provide statistics on bandwidth, message rate, etc. If the messages are marshalled according to a formal type system, then a traffic inspection module could automatically decode messages in much the same way a program debugger can automatically decode stack variables of a running application.

LCM provide tools for marshalling, communication, and analysis that are both simple to use and highly efficient. Its overarching design philosophy is to make it easy to accomplish the most common message passing tasks, and possible to accomplish most others. LCM also detects many run-time errors, such as invalidly formatted data and type mismatches.

\section{RElATED WORK}

Interprocess communication is an extensively studied topic with broad applicability. We direct our attention specifically towards its use in developing robotic systems, where the idea of dividing large systems into modules has become commonplace [2], [3].

There are several recurring themes in existing systems. Publish/subscribe models are the most commonly used [4], [2], [5], with TCP being the most common transport. Most of these systems employ a centralized hub, whether it is used for message routing or merely for "match making". Virtually all of these systems provide a reliable and ordered transport system, though some of the systems provide a UDP transport as a non-standard option. A separate technical report describes a number of commonly used systems in greater detail [6]. 
Existing systems are widely varied in terms of provided support for data marshalling. Some systems do not provide automatic marshalling tools, instead allowing free-form human-readable messages [4], or specifying a set of standardized messages and binary formats [7]. Several systems use an XDR-based marshalling system [8], [9], [2], though some implementations provide only partially automatic code generation. Language and platform support is typically limited, and with some systems [2], the users must manually keep a formatting string in sync with the message layout.

Our system, Lightweight Communications and Marshalling (LCM), provides a "push"-based publish/subscribe model. It uses UDP multicast as a low-latency but unreliable transport, thus avoiding the need for a centralized hub. LCM provides tools for generating marshalling code based on a formal type declaration language; this code can be generated for a large number of platforms and operating systems and provides run-time type safety.

Several of the other systems provide an operating "environment", consisting of pre-defined data types, ready-touse modules, event loops, message-passing systems, visualization and simulation tools, package management, and more [2], [3], [4]. LCM is different in that it is intended to be an "a la carte" message passing system, capable of being integrated into a wide variety of systems.

Finally, the way in which LCM is perhaps most distinctive from other systems is in its emphasis on debugging and analysis. For example, while all systems provide some mechanism for delivering a message from one module to another, few provide a way to easily debug and inspect the actual messages transmitted. Those that do typically do so at the expense of efficiency and performance. LCM provides a tool for deep inspection of all messages passed on the network, requiring minimal developer effort and incurring no performance penalty. This is made possible by design, and allows a system developer to quickly and efficiently identify many bugs and potential sources of failure that are otherwise difficult to detect.

\section{APPROACH}

We divide our description of LCM into several sections: type specification, marshalling, communications, and tools. Type specification refers to the method and syntax for defining compound data types, the sole means of data interchange between processes using LCM. Marshalling is the process of encoding and decoding such a message into binary data for the communications system which is responsible for actually transmitting it.

\section{A. Type Specification}

Processes communicating with LCM agree in advance on the compound data types used to represent information. LCM does not support defining Remote Procedure Calls (RPC), and instead requires applications to communicate by exchanging individual messages. This restriction makes the LCM messaging protocol stateless, simplifying other aspects of the system (particularly logging and playback). To aid this process, LCM defines a formal type specification language for describing messages.

The binary representation of a message is implicitly defined by its type definition. These definitions are independent of platform and programming language, and a code generation tool is used to automatically generate language-specific bindings that provide representations of the message in a form native to the programming language. Fig. 1 shows an example of two LCM message type definitions, and Fig. 2 demonstrates use of the $\mathrm{C}$ language bindings.

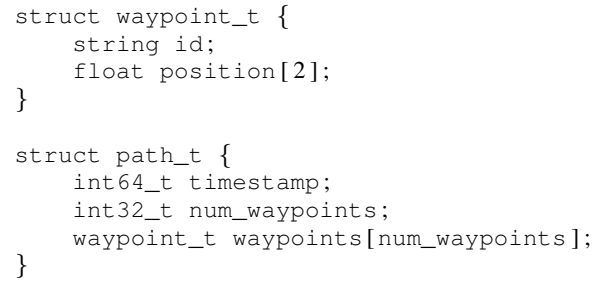

Fig. 1. Two example LCM types. The first contains two fields, one of which is a fixed-length array. The second is a compound type, and contains a variable length array in addition to two primitive data types.

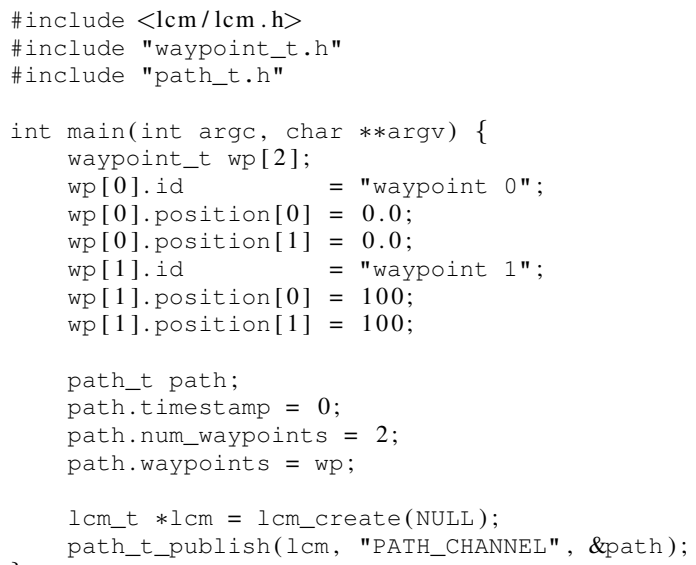

Fig. 2. A complete $\mathrm{C}$ program showing how to declare and transmit a message using the automatically generated $\mathrm{C}$-language bindings for the types defined in Fig. 1. LCM also supports message type bindings for Python, Java, and MATLAB.

The types in Fig. 1 show the basic structure of LCM types. The LCM documentation and a separate technical report [6] contain a comprehensive description of the type specification language and additional features such as namespaces and constants.

The LCM type specification was strongly influenced by the External Data Representation (XDR) [8], which is used by Sun Remote Procedure Calls (Sun/RPC) and the Network File System (NFS) [10]. Some XDR features, such as pointer chasing and unions, are not supported by LCM due to the fact that they are rarely used, have portability issues, or invite user error.

LCM also provides features and other usability improvements over XDR. For example, LCM provides a simple method for declaring the length of a variable-length array; in contrast, the XDR specification does not specify how the 
length of arrays should be specified, which has led to a variety of incompatible approaches. A second example is LCM's support for namespaces, which make it easier to avoid naming conflicts when sharing code with others.

\section{B. Marshalling}

In order for two modules to successfully communicate, they must agree exactly on how to interpret the binary contents of a message. If the interpretations are different, the resulting system behavior is typically undefined, and usually unwanted. In some cases, these problems can be obvious and catastrophic: a disagreement in the signedness of a motor control message, for example, could cause the robot to suddenly jump to maximum reverse power when the value transitions from $0 \times 7 f$ to $0 \times 80$. In other cases, problems can be more subtle and difficult to diagnose.

Additionally, as a system evolves, the messages may change as new information is required and obsolete information is removed. Thus, message interpretation must be synchronized across modules as messages are updated.

Fingerprint: Type checking in LCM is accomplished by prepending each LCM message with a 64-bit fingerprint derived from the type definition. The fingerprint is a hash of the member variable names and types. If the LCM type contains member variables that are themselves LCM types, the hash recursively considers those member variables. The details of computing a hash function are straight-forward and thoroughly documented within the LCM source code distribution, so we omit a detailed description here.

In the common case, an LCM client knows what type of message is expected on a particular messaging channel. When a message is received by an LCM client, it first reads the fingerprint of the message. If the fingerprint does not match the LCM client's expected fingerprint, a type error is reported.

LCM clients can also build a fingerprint database, allowing them to identify the type of message when it is received. This is the technique used by our tool lcm-spy, which allows realtime deep inspection of LCM traffic.

\section{Communications}

The communications aspect of LCM can be summarized as a publish-subscribe messaging system that uses UDP multicast as its underlying transport layer. Under the publishsubscribe model, each message is transmitted on a named channel, and modules subscribe to the channels required to complete their designated tasks. It is typically the case (though not enforced by LCM) that all the messages on a particular channel are of a single pre-specified type.

1) UDP Multicast: In typical publish-subscribe systems, a mediator process is used to maintain a central registry of all publishers, channels, and subscribers. Messages are then either routed through the mediator directly, or the mediator is used to broker point-to-point connections between a publisher and each of its subscribers. In both cases, the number of times a message is actually transmitted scales linearly with

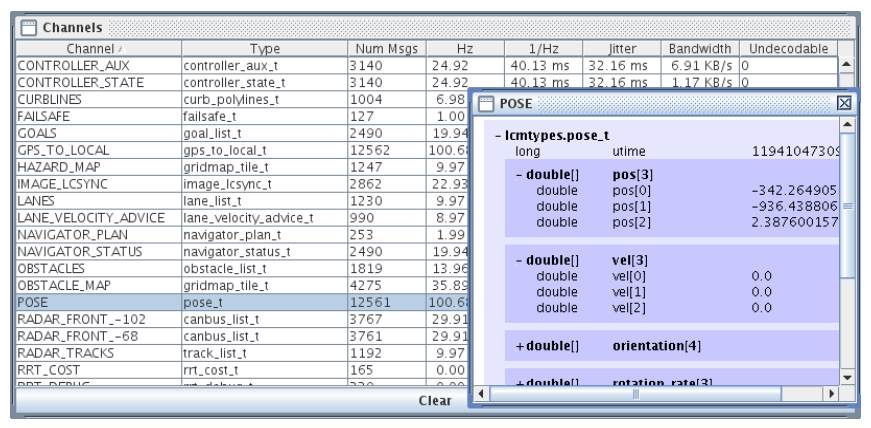

Fig. 3. LCM-spy screenshot. lcm-spy is a traffic inspection tool that is able to automatically decode and display LCM messages in real-time. It requires only that the automatically generated Java code for the LCM types be accessible in the class path; no additional developer effort is required.

the number of subscribers. When a message has multiple subscribers, this overhead can become substantial.

The approach taken by LCM, in contrast, is simply to broadcast all messages to all clients. A client discards those messages to which it is not subscribed. Communication networks such as Ethernet and the 802.11 wireless standards make this an efficient operation, where transmitted packets are received by all devices regardless of destination.

LCM bases its communications directly on UDP multicast, which provides a standardized way to leverage this feature. Consequently, it does not require a centralized hub for either relaying messages or for "match making". A maximum LCM message size of $4 \mathrm{~GB}$ is achieved via a simple fragmentation and reassembly protocol. The multicast time-to-live parameter is used to control the scope of a network, and is most commonly set to 0 (all software modules hosted on the same computational device) or 1 (modules spread across devices on the same physical network).

2) Delivery Semantics: LCM provides a best-effort packet delivery mechanism and gives strong preference to the expedient delivery of recent messages, with the notion that the additional latency and complexity introduced by retransmission of lost packets does not justify delaying newly transmitted messages.

In general, a system that has significant real-time constraints, such as a robot, may often prefer that a lost packet (e.g., a wheel encoder reading) simply be dropped rather than delay future messages. LCM reflects this in its default mode of operation; higher level semantics may still be implemented on top of the LCM message passing service.

\section{Tools}

LCM provides several tools useful for logging, replaying, and inspecting traffic (Fig. 3). Together, they allow a developer to rapidly and efficiently analyze the behavior and performance of an LCM system.

The logging tools are similar to those found in many messaging systems, and allow LCM traffic to be recorded to a file for future playback or analysis. We note that the logging and playback programs are not attributed special status in 

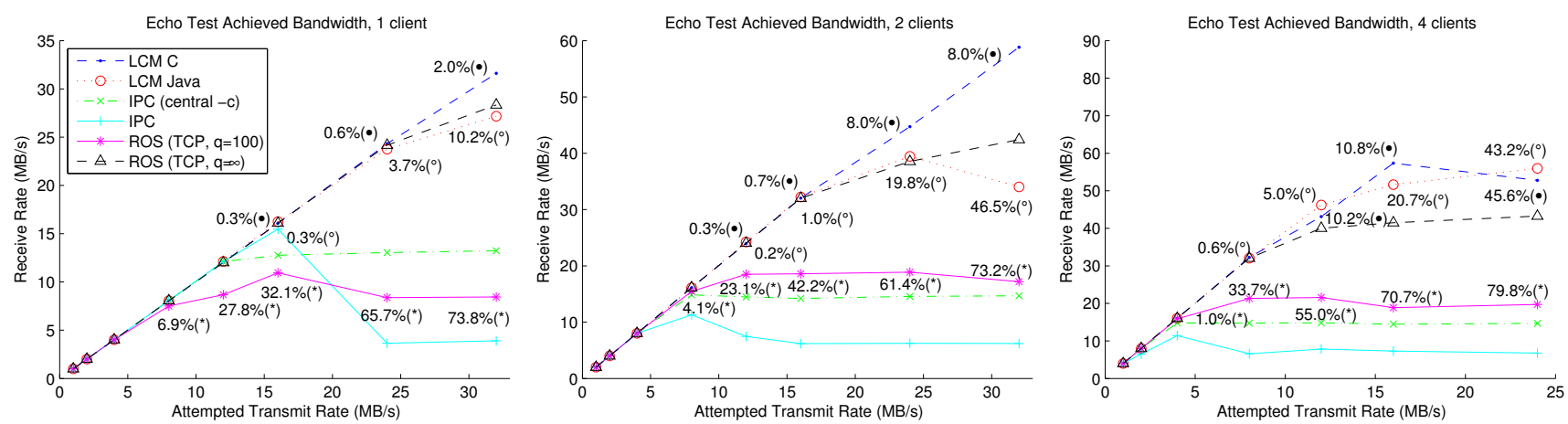

Fig. 4. Bandwidth results from an echo test with 1, 2, and 4 clients, where each client echoes messages transmitted by a single sender. The bandwidth of successful echoes as detected by the original sender are shown. Message loss rates (messages with no received echo) are shown in parentheses when nonzero.
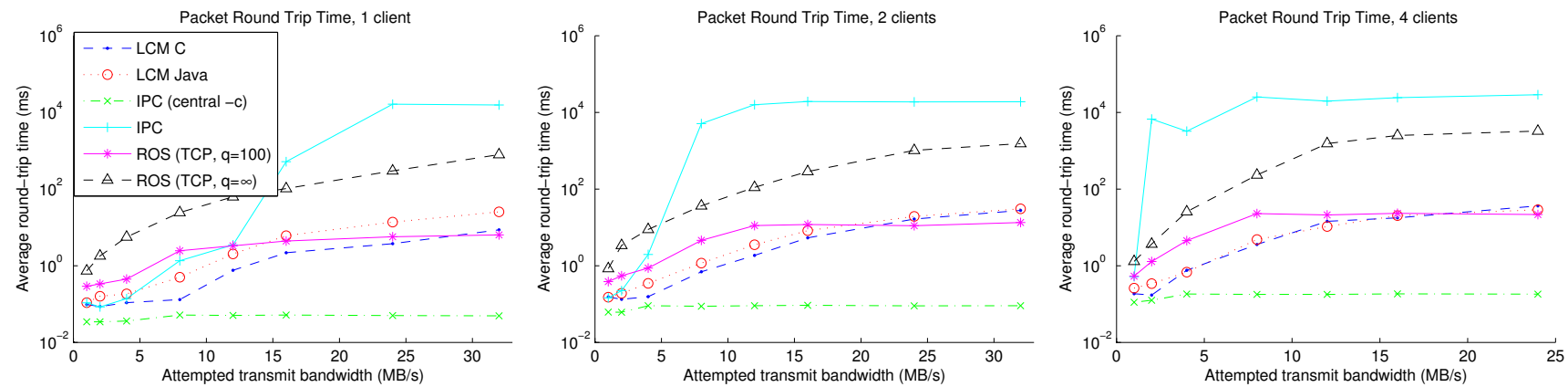

Fig. 5. Mean message round-trip times for the echo test in Fig. 4 with 1,2, and 4 clients. Average round-trip times are shown on a log scale; these times do not reflect lost packets (which essentially have an infinite round-trip time). Both LCM implementations offer low latency. While IPC (using central -c) also appears to provide low latency, it is critical to notice that IPC's achieved bandwidth fell short of the target bandwidth (see Fig. 4).

LCM; the logger simply subscribes to all channels, and the replay tool publishes messages from a data file.

Spy: If a database of every LCM type used on a system is assembled, then an arbitrary fingerprint serves as a type identifier with very high probability. ${ }^{1}$ The $1 \mathrm{~cm}$-spy tool is designed to leverage this attribute, and is able to analyze, decode, and display live traffic automatically with virtually no programmer effort. Fig. 3 shows a screenshot of lcm-spy inspecting traffic.

lcm-spy is implemented in Java, and requires only that the classpath contain the automatically generated Java versions of each type. Using the reflection features of Java, it searches the classpath for classes representing LCM types, building a mapping of fingerprints to LCM types. Because each field of an LCM message is strongly typed, lcm-spy is able to automatically determine a suitable way to decode and display each field of a message as it is received.

lcm-spy also provides summary statistics for message channels such as message rate, number of messages counted, and bandwidth utilized. Together, these features provide a highly useful view of an LCM network.

When used in practice, 1cm-spy allows developers to

\footnotetext{
${ }^{1}$ The fingerprints of each LCM type are represented as 64 bit integers, providing theoretical collision resistance for $2^{32}$ different types. While LCM's non-cryptographic hash function degrades this figure, the probability of collisions is vanishingly small for the few hundred message types that a large system might employ.
}

quickly identify many of the most common failures. During module development, it can help verify that a module is producing messages on the correct channels at the expected rate and bandwidth. It can also be used to inspect arbitrary messages to check the values of any field - useful for tracking down bugs and verifying module operation.

In our experience, lcm-spy is a critically important tool, on par with program debuggers and profilers. Whereas a debugger is useful for inspecting the internal state of a module, lcm-spy has become invaluable for inspecting messages passed between modules. Because it passively observes and analyzes traffic, lcm-spy can provide this insight with absolutely no impact on the system performance.

\section{PERFORMANCE}

One way to measure the interprocess communication performance of LCM is by examining its bandwidth, latency, and message loss under various conditions. Figs. 4 and 5 show a comparison of the $\mathrm{C}$ and Java implementations of LCM with IPC and the ROS TCP transport.

In this test, a source node transmits fixed-size messages at various rates. One, two, or four client nodes subscribe to these messages and immediately retransmit (echo) them once received. The source node measures how many messages are successfully echoed, the round-trip time for each echoed message, and the bandwidth consumed by the original transmission and the echoes. For this test, IPC was run in two 
modes, one in which the central dispatching server relays all data (the IPC default), and another in which the central server acts merely as a "match maker" facilitating peer-topeer connections (central -C). To improve performance, operating system send and receive buffers were increased to $2 \mathrm{MB}$. The ROS test was implemented using ROS Box Turtle, with TCP_NODELAY set and two different maximum queue lengths to trade off message loss and latency.

To collect each data point, the sender transmits $100 \mathrm{MB}$ of data split into 800 byte messages at a fixed rate determined by a target transmission bandwidth. Figs. 4 and 5 show the results for tests conducted on an eight-core workstation running Ubuntu 9.04. Hosting each process on separate identical workstations connected via 1000Base-T Ethernet yielded similar results. In some cases, the actual message transmission rate does not match the target transmission rate due to transport and software limitations.

From these figures, we can see that LCM scales with both the amount of traffic to be sent and the number of subscribers. As network capacities are reached, LCM minimizes latency and maintains high bandwidth by dropping messages. The LCM Java implementation performs comparably to the $\mathrm{C}$ implementation, and responds to computational limits of the virtual machine by dropping more messages.

IPC performs well with one subscriber, but does not scale as well to multiple subscribers as a result of transmitting multiple copies of each message. ${ }^{2}$ Using the match-making service of IPC (central $-c$ ) improves bandwidth and reduces latency, but ultimately has the same difficulties. We note that although IPC with peer-to-peer connections maintains low latency as the attempted transmission rate is increased, the actual bandwidth achieved does not increase due to link saturation from duplicated messages. For example, with four clients echoing messages, IPC is unable to transmit faster than $11 \mathrm{MB} / \mathrm{s}$, as the bandwidth consumed by the quadruplicate transmission and the echoes saturates the link capacity.

The ROS TCP transport achieves high throughput when the maximum queue length $(q)$ is set to infinity, but at the expense of message latency. Various settings of $q$ provide an adjustable tradeoff between throughput and latency. With $q=100$, message latency is on par with LCM at higher data rates, but message loss is more frequent due to filled buffers.

\section{A. Marshalling Performance}

In addition to performance of the communications system, we are also interested in the computational efficiency of the LCM marshalling and unmarshalling implementations. Performance of a marshalling system is a function of the complexity and nature of the message, and the presence of arrays, nested types, strings, and other fields are all factors. We compared the performance of LCM, IPC, and ROS on

\footnotetext{
${ }^{2}$ Our initial experiments with IPC, using Carmen 0.7.4-beta, produced much worse results due to coarse-grained timing functions in IPC that degrade performance when packet rates exceed $\sim 1 \mathrm{kHz}$. We improved this performance by exporting higher-resolution versions of those functions; this improved data is used in this paper.
}

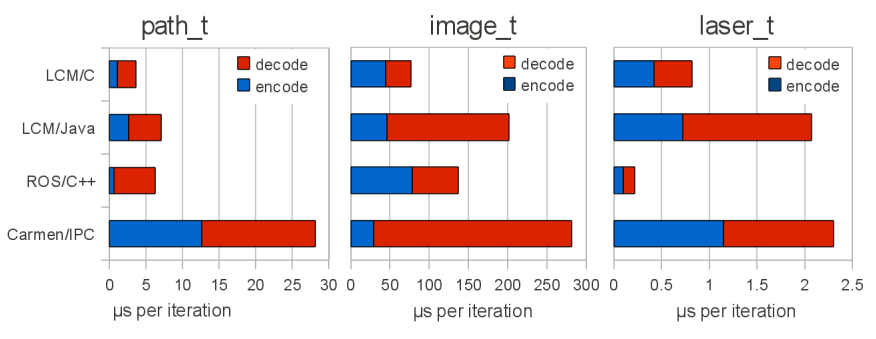

Fig. 6. Marshalling performance for four different marshalling implementations on three different types. Smaller numbers are better.

three common message types - a $640 \times 480$ grayscale camera image, a single scan of a planar laser range scanner with 180 range measurements, and a list of 50 waypoints. The LCM types in Fig. 1 are used for the waypoint list; the LCM types for the image and laser scan messages are:

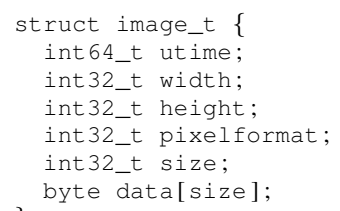

Similar message types were defined for IPC and ROS. In this test, we estimate the amount of time each implementation spends encoding and decoding a single message by averaging over the time taken to encode and decode $10^{6}$ messages. Estimates were taken 10 times and then averaged. Timings are shown in Fig. 6.

The LCM C implementation was the fastest on the path $\_t$ and image $\_t$ messages, and the ROS $\mathrm{C}++$ implementation was the fastest on the laser_t message. The little-endian assumption made by ROS yields faster performance when handling messages with many multi-byte primitive types on little-endian computers. The pure Java LCM implementation is generally competitive but slower handling endianness (laser_t $\mathrm{t}$ ) and large memory allocations (image_t). Carmen, despite being written in $\mathrm{C}$, is fairly slow due to not using faster block copy operations when possible.

Finally, it should be noted that the communication and marshalling comparisons given here consider only a few common scenarios. Performance may vary with packet size, transmit rate, message structure, etc. Each messaging implementation can often be adjusted to various degrees, based on the messaging and system requirements. However, these comparisons do serve to provide some insight into the overall performance of each system.

\section{Case Studies}

Since its development, LCM has been used as the primary communications system for a number of robotics research platforms operating in real environments. Here, we describe some examples of how LCM was used to assist the development of a robotics research system. 


\section{A. Urban Challenge}

The 2007 DARPA Urban Challenge was an autonomous vehicle race designed to stimulate research and public interest in autonomous land vehicle technology. Vehicles were required to safely navigate a $90 \mathrm{~km}$ race course through a suburban environment in the presence of both robotic- and human-driven vehicles. LCM served as the communications backbone for the Ford/IVS and MIT teams [11]. On the MIT vehicle, 70 separate modules were simultaneously active, spread across 10 networked workstations. The average bandwidth used by the entire LCM network was $16.6 \mathrm{MB} / \mathrm{s}$, with an average transmission rate of 6,775 messages/s.

Messages ranged from very small updates to camera images and obstacle maps up to several megabytes in size. Some, such as the pose estimates, were subscribed to by virtually every module on the network, while others had only one or two subscribers.

Throughout the development process, almost 100 different message types were used, many of which changed frequently as the capabilities and requirements of each module evolved. Software development was distributed across many people working from different locations, and the LCM type definitions became a convenient place for developers to agree on how modules would interface.

Because language-specific bindings could be generated automatically from LCM type definitions, modifying messages and the modules that used them to add or remove fields could often be accomplished in the span of minutes. Additionally, the runtime type checking of LCM fingerprints provided a fast and reliable way to detect modules that had not been recompiled to use the newly modified form of a message.

\section{B. Land, Underwater, and Aerial Robots}

Since the Urban Challenge, LCM has been applied to a number of other robot research platforms such as small indoor wheeled robots, arm manipulators, quadrotor helicopters [12], and an autonomous forklift [13]. In many cases, modules used in one system were easily transitioned to others by ensuring that the LCM messages they needed for correct operation were present on the target robot.

In one underwater multi-vehicle research project [14], each vehicle contains on-board sensors, thrusters, and a computer for data processing and vehicle control. Despite a significantly different application domain from the Urban Challenge, the software engineering principles remain identical, and LCM has proved just as useful. New message types are easily defined as needed, and software modules are adapted to operate in different domains.

\section{CONCLUSION}

We have presented LCM and its design principles. LCM is driven by an emphasis on simplicity and a focus on the entire development process of a robotic software system. In addition to achieving high performance, LCM also provides tools for traffic inspection and analysis that give a developer powerful and convenient insight into the state of the robotic system.

The LCM type specification language is designed to allow flexible and intuitive descriptions of a wide class of data structures. Type fingerprints allow for runtime type checking and identification, and automatically generated language bindings result in a simple and consistent API for manipulating messages and the data they represent. Native support for multiple platforms and languages allows developers to choose the environment most suitable for the task at hand.

LCM has been used as the core communications infrastructure on a number of demanding robotic systems on land, water, and air. In each of these cases, the simplicity and versatility of LCM allowed for rapid development of complex software systems. The modular nature of these systems has allowed for significant code re-usability and application of modules developed from one system to another.

LCM is distributed at http://1 cm.googlecode. com. It is supported on Microsoft Windows XP/Vista/7 and all POSIX.1-2001 compliant platforms (GNU/Linux, OS/X, FreeBSD, etc.)

\section{REFERENCES}

[1] D. L. Parnas, "On the criteria to be used in decomposing systems into modules," Commun. ACM, vol. 15, no. 12, pp. 1053-1058, 1972.

[2] M. Montemerlo, N. Roy, and S. Thrun, "Perspectives on standardization in mobile robot programming: The carnegie mellon navigation (carmen) toolkit," in Proc. Int. Conf. on Intelligent Robots and Systems, vol. 3, Las Vegas, NV, USA, October 2003, pp. 2436-2441.

[3] Willow Garage, "Robot Operating System," http://www.ros.org.

[4] P. M. Newman, "MOOS - mission orientated operating suite," Massachusetts Institute of Technology, Tech. Rep. 2299/08, 2008.

[5] R. Simmons and D. James, Inter-Process Communication, Aug. 2001.

[6] A. S. Huang, E. Olson, and D. Moore, "Lightweight communications and marshalling for low latency interprocess communication," Massachusetts Institute of Technology, Tech. Rep. MIT-CSAIL-TR-2009041, 2009.

[7] J. W. Group, The Joint Architecture for Unmanned Systems: Reference Architecture Specification, June 2007.

[8] R. Srinivasan, "XDR: external data representation standard," http://www.rfc-editor.org/rfc/rfc1832.txt, Internet Engineering Task Force, RFC 1832, Aug. 1995.

[9] T. H. Collet, B. A. MacDonald, and B. P. Gerkey, "Player 2.0: Toward a practical robot programming framework," in Proc. Australasian Conf. on Robotics and Automation, Sydney, Australia, Dec. 2005.

[10] Sun Microsystems, "NFS: network file system protocol specification," http://www.rfc-editor.org/rfc/rfc1094.txt, Internet Engineering Task Force, RFC 1094, Mar. 1989.

[11] J. Leonard et al., "A perception-driven autonomous vehicle," Journal of Field Robotics, vol. 25, no. 10, pp. 727-774, Oct 2008.

[12] M. Achtelik, A. Bachrach, R. He, S. Prentice, and N. Roy, "Stereo vision and laser odometry for autonomous helicopters in GPS-denied indoor environments," G. R. Gerhart, D. W. Gage, and C. M. Shoemaker, Eds., vol. 7332, no. 1. SPIE, 2009, p. 733219.

[13] S. Teller et al., "A voice-commandable robotic forklift working alongside humans in minimally-prepared outdoor environments," in Proc. Int. Conf. Robotics and Automation, Anchorage, AL, USA, May 2010.

[14] H. C. Brown, A. Kim, and R. M. Eustice, "An overview of autonomous underwater vehicle research and testbed at PeRL," Marine Technology Society Journal, 2009. 